\title{
Comprehensive Approach for Mathematical Modeling of Mechanical Systems: Fixture Design Case Study
}

\author{
Vitalii Ivanov ${ }^{1}$, Ivan Pavlenko² \\ ${ }^{1,2}$ Sumy State University, Faculty of Technical Systems and Energy Efficient Technologies, \\ 2 Rymskogo-Korsakova St., Sumy, 40007, Ukraine \\ ivanov@tmvi.sumdu.edu.ual, i.pavlenko@omdm.sumdu.edu.ua ${ }^{2}$
}

\begin{abstract}
Present work is devoted to locating and clamping of prismatic parts in fixtures during the machining on drilling-milling-boring machines. The special feature of locating schemes of parts of the present type is that one of the flat surfaces is used as a datum ( 3 contact points) ensuring stability, convenience of locating and vibration elimination during the machining process. Within the scope of the presented work, the comprehensive mathematical model of the system "fixture - workpiece" has been developed taking into account technological features of locating and clamping processes of workpiece in fixture. The methodology of determination of the rigidity of the cutting tool and functional elements has been proposed. As a result, the static and dynamic analysis of the workpiece in the fixture under the effect of spatial system of cutting and clamping forces is being determined. In addition, the free frequencies of the system "fixture-workpiece" and forms of the free and forced vibrations, frequency-response characteristics are being determined.
\end{abstract}

Keywords: locating chart, functional element, rigidity, free vibrations, free frequency, forced vibrations, cutting force, frequency-response characteristic.

\section{Introduction}

Fixtures are used for ensuring the precise locating and reliable clamping of workpieces during the machining on the metal-cutting machines and are an integral part of the closed-loop technological system "machine tool - fixture - cutting tool - workpiece". In particular, fixtures considerably effect on the output of the competitive products, ensuring the steady state accuracy and quality of the work surfaces, sufficient rigidity of the technological system, flexibility of production and initial cost of the final product. It is definitely confirmed by the percentage number of fixtures that makes $70-80 \%$ from the total quantity of tooling [1]; 10-20\% of the total cost of manufacturing system [2]; 80-90\% of the total time for production planning [3]; $40 \%$ of the rejected parts after the machining due to imperfection fixture design [4]; 70\% of new fixtures are the modification of the existing [5].

Besides, the majority of existing methods of calculation do not include the dynamic character of the cutting process and interaction of the workpiece with the elements of fixture. Therefore, the problem of designing of fixtures is very urgent and is connected 
with the basic multidisciplinary scientific and practical tasks on ensuring the stable locating of workpiece in the fixture during the machining process of the surfaces of parts.

\section{The current state of researches in fixture design}

At present, different approaches to the designing and research of fixture for carrying out the manufacturing operations of machining of parts of different types are being developed. With the purpose of determining of the present state of researches in fixture designing and determining of the urgency of the research, the basic approaches to the designing of fixture from the point of view of interaction between fixture and workpiece have been considered.

In the work [6] friction between elements of fixture and workpiece has been researched and have been determined the deformations, which appear in the points of contact. The authors of the article [7] developed the methodology of modeling of the system "fixture - workpiece" and determined the influence of the previous load from the impact of clamping and cutting forces on the error of the work surface. In work [8] the methodology of analysis of stability of the system "fixture - workpiece" has been developed and calculation of the minimum clamping force, required for machining process, has been presented; also, the influence of sequence of workpiece clamping has been researched. J. Asante analytically calculated and researched the influence of modes of cutting and fixture compliance on the workpiece stability [9]. Work [10] presents the simplified analytical model of the contact interaction between clamping elements of fixture and workpiece and the developed finite element model allows evaluate the contact deformation in contact points between clamping elements and workpiece. Y. Zheng developed the finite element model of determine the stability of workpiece locating in fixture, and developed the methodology of optimization of the previous loading [11]. In work [12] the authors developed the mathematical model of fixture interaction with workpiece and analysis of deterministic positioning of fixture. The authors Y. Rong and Y. Bai have a number of researches in the area of fixture accuracy and analysis of the fixture stability that is based on the consideration of two-dimensional problem and introduction of "operative factor", which takes into account the friction forces [13]. In work [14] the methodology on determination of points and forces of clamping for ensuring the stable locating of workpiece in fixture has been developed. Researches [15] are devoted to the developing of the methodology of control of clamping force taking into account the contact interaction between workpiece and clamping elements by means of the methods of nonlinear programming. In work [16] the problem of the temporariness of the stability of fixture locating taking into account the restriction of the force and direction of its action in the system "fixture - workpiece" has been researched. H. Deng as opposed to quasi-static approach of predecessors considered the influence of material removal on the dynamic state of the system "fixture - workpiece" [17]. The authors of the article [18] researched the displacement of the workpiece because of the cutting forces while locating on the plane under condition of the known coefficient of rigidity of the functional elements. An essential experience of research of mechanical system "fixture - workpiece" has been obtained in terms of ensuring the 
condition of workpiece stability in fixture [19], [20], determine of free vibrations [21], determine of forced vibrations [22], [23], analysis of rigidity of functional elements [24], development of software environments of the numerous realization of algorithms of the calculation of statistic and dynamic analysis [25].

Considering that the existing information on determine the contact points of functional elements of fixture, which are locating and clamping elements, is of advisory character and do not base on the analytical researches; and basing on that fact that the existing methodologies of calculation of clamping forces do not include the condition of stability of workpiece and provide the unreasonably overestimated value, performing of the researches is the relevant objective.

The present work is focused on locating and clamping of prismatic parts fixtures during the machining on drilling-milling-boring machines. Setup of prismatic parts in fixtures is performed according to 3 basic locating charts (according to three planes, according to two planes and a hole, according to plane and two holes), which make $76 \%$ from all locating charts of the parts of this type. The characteristic feature of these schemes is that one of the flat surfaces is used as a datum ( 3 contact points), ensuring the stability, convenience of locating and preventing of vibrations during the machining [26].

The goal of the proposed work is the development of the comprehensive mathematical model of the system "fixture - workpiece" in view on the technological features of the process of locating and clamping of the workpiece in the fixture taking into account statistic and dynamic analysis of the workpiece in fixture under the effect of spatial system of cutting and clamping forces.

To achieve the set objectives the following tasks should be solved:

- static analysis of the system "fixture - workpiece";

- research of the free frequencies of the system and determine of the correspondence forms of free vibrations;

- research of the forced vibrations of the system and determine of the frequency-response characteristics;

- development of the methodology of calculation of the system and development of the practical recommendations concerning its use during fixture design.

\section{Static analysis of the system "fixture - workpiece"}

\subsection{Determination of the static reactions in the fixture functional elements}

Consider the conservative mechanical system, which consists from workpiece, clamped in 6 supports $1,2, \ldots, 6$ in the global orthogonal coordinate system XYZ with the center in the point $\mathrm{O}$ (Fig. 1).

External active forces are:

- components of the cutting force $\mathrm{F}_{\mathrm{x}}, \mathrm{F}_{\mathrm{y}}$ and $\mathrm{F}_{\mathrm{z}}$, applied in the point $\mathrm{A}\left(\mathrm{X}, \mathrm{Y}, \mathrm{H}_{1}\right)$;

- clamping force $\mathrm{Q}$, applied in the point $\mathrm{B}(\mathrm{L} / 2, \mathrm{~B} / 2, \mathrm{H})$;

- weight of the workpiece $\mathrm{G}$, applied in the weight center $\mathrm{C}\left(\mathrm{X}_{\mathrm{C}}, \mathrm{Y}_{\mathrm{C}}, \mathrm{Z}_{\mathrm{C}}\right)$. 

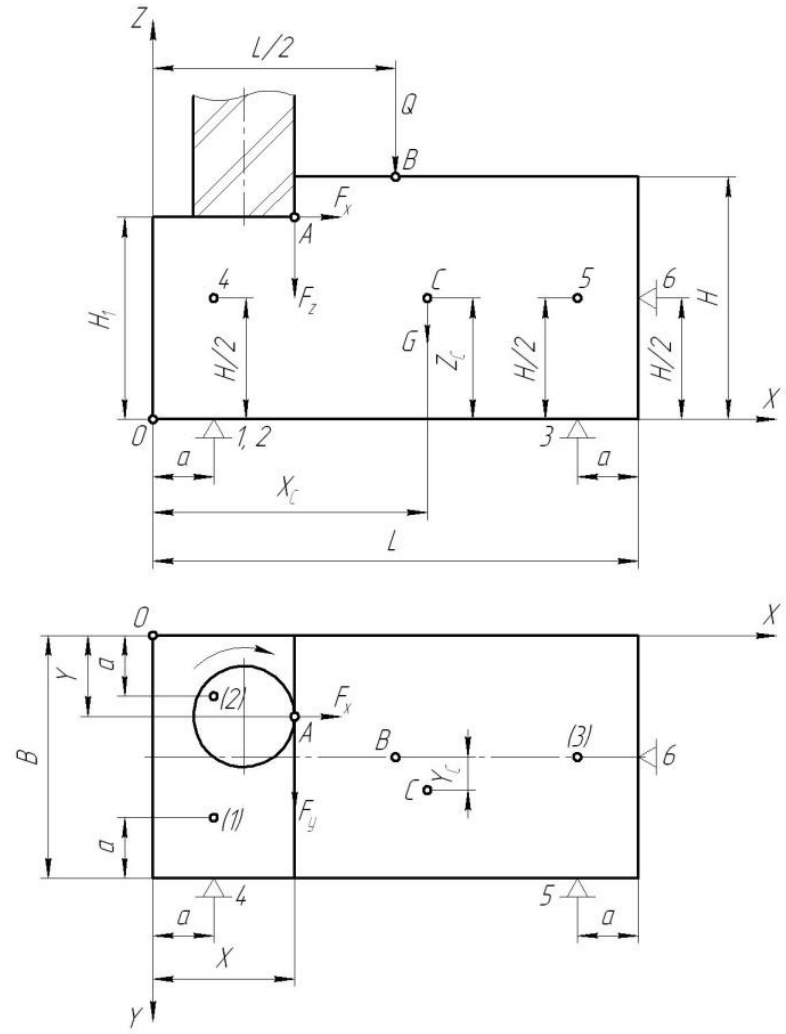

Fig. 1. Calculation scheme of the mechanical system "fixture - workpiece".

Following the principle of the release the constraints, the action of the supporting surfaces on the workpiece is replaced by 6 reactions of supports $R_{1}, R_{2}, \ldots, R_{6}$. Equilibrium conditions of workpiece in the fixture for the spatial force system have the following view:

$$
\left\{\begin{array}{l}
F_{x}-R_{6}=0 ; \quad F_{y}-R_{4}-R_{5}=0 ; \quad-F_{z}-Q-G+R_{1}+R_{2}+R_{3}=0 ; \\
F_{y} H_{1}+F_{z} Y+Q B / 2+G Y_{C}-R_{1}(B-a)-R_{2} a-R_{3} B / 2-R_{4} H / 2-R_{5} H / 2=0 ; \\
-F_{x} H_{1}-F_{z} X-Q L / 2-G X_{C}+R_{1} a+R_{2} a+R_{3}(L-a)+R_{6} H / 2=0 ; \\
F_{x} Y-F_{y} X+R_{4} a+R_{5}(L-a)-R_{6} B / 2=0 .
\end{array}\right.
$$

The obtained system of six linear algebraic equations with six unknown reactions $R_{i}$ $(\mathrm{i}=1,2, \ldots, 6)$ in a matrix form is as follows:

$$
[A]\{R\}=\{F\}+\{Q\}+\{G\},
$$

where $[\mathrm{A}]$ - coefficient matrix (directional cosines and axle arm): 


$$
[A]=\left[\begin{array}{cccccc}
0 & 0 & 0 & 0 & 0 & 1 \\
0 & 0 & 0 & 1 & 1 & 0 \\
1 & 1 & 1 & 0 & 0 & 0 \\
B-a & a & B / 2 & H / 2 & H / 2 & 0 \\
a & a & L-a & 0 & 0 & H / 2 \\
0 & 0 & 0 & a & L-a & -B / 2
\end{array}\right]
$$

$\{\mathrm{F}\},\{\mathrm{Q}\},\{\mathrm{G}\}$ - column-vectors of cutting force, clamping force and gravity force:

$$
\{F\}=\left\{\begin{array}{c}
F_{x} \\
F_{y} \\
F_{z} \\
F_{y} H_{1}+F_{z} Y \\
F_{x} H_{1}+F_{z} X \\
-F_{x} Y+F_{y} X
\end{array}\right\} ;\{Q\}=\left\{\begin{array}{c}
0 \\
0 \\
Q \\
Q B / 2 \\
Q L / 2 \\
0
\end{array}\right\} ;\{G\}=\left\{\begin{array}{c}
0 \\
0 \\
G \\
G Y_{C} \\
G X_{C} \\
0
\end{array}\right\} .
$$

Vector-column of the unknown reactions of supports is determined from the equation (2) by means of the methodology of the inverted matrix:

$$
\{R\}=[A]^{-1}(\{F\}+\{Q\}+\{G\}) .
$$

Under condition of invertible matrix $[\mathrm{A}]$ :

$$
\operatorname{det}[A]=(2 a-L)^{2}(2 a-B) \neq 0,
$$

which corresponds the simultaneous performing of both conditions: $\mathrm{a} \neq \mathrm{L} / 2, \mathrm{a} \neq \mathrm{B} / 2$.

Elements of the vector-column $\{R\}$ are forces of reactions of the functional elements as a result of influence of the forces of cutting, clamping and gravity. According to the theory of parts locating, the assignment of the positions of functional elements during the locating must ensure the stable equilibrium locating of the workpiece. As the locating chart includes the supports of single action and the case of negative meanings of the reactions points to the absent of the contact between workpiece and the correspondent functional element of the fixture, so the checking of the conditions of loading of supports should be carried out:

$$
R_{i}>0 \quad(i=1,2, \ldots, 6) .
$$

Analysis of the solution (5) shows that the necessary and sufficient conditions of the inequality (7) are geometric and physical constraints: 


$$
\begin{cases}a<\frac{\min \{B ; L\}}{2} ; & a<X<\frac{L}{2} ; \quad H_{1}>\frac{H}{2} \\ \frac{F_{x}}{F_{y}}<\frac{2(X-a)}{B} ; & Q>\frac{B+2 a}{B-2 a} F_{z} .\end{cases}
$$

\subsection{Determination of the rigidity coefficients of the fixture functional elements}

Static loads $R_{i}$ on the compliant supports $1,2, \ldots, 6$ cause their deformation. The corresponding values of displacements of the points of the supporting surface may be defined by the empirical dependence:

$$
s_{i}=c_{i} R_{i}^{n_{i}},
$$

which could be obtained by means of approximation of the existing experimental data of physical or virtual experiments.

Herewith, the approximation procedure is carried out according to two parameters: $c_{i}-$ is the compliance coefficient of support; $n_{i}-$ an exponent. The corresponding regression model is based on the method of minimizing of the total quadratic error $\Delta_{\mathrm{i}}$, obtained after the previous logarithm equation (9). The method of least squares

$$
\Delta_{i}=\sum_{j=1}^{N}\left(\ln c_{i}+n_{i} \ln R_{i}^{\langle j\rangle}-\ln s_{i}^{\langle j\rangle}\right)^{2} \rightarrow \min ,
$$

allows determining the parameters $\mathrm{c}_{\mathrm{i}}, \mathrm{n}_{\mathrm{i}}$ from the following conditions:

$$
\frac{\partial \Delta_{i}}{\partial\left(\ln c_{i}\right)}=0 ; \quad \frac{\partial \Delta_{i}}{\partial n_{i}}=0
$$

which are reduced to a system of two equations:

$$
\left\{\begin{array}{c}
\ln c_{i} \\
n_{i}
\end{array}\right\}=\left[\begin{array}{cc}
N & \sum_{j=1}^{N} \ln R_{i}^{\langle j\rangle} \\
\sum_{j=1}^{N} \ln R_{i}^{\langle j\rangle} & \sum_{j=1}^{N} \ln ^{2} R_{i}^{\langle j\rangle}
\end{array}\right]^{-1}\left\{\begin{array}{c}
\sum_{j=1}^{N} \ln s_{i}^{\langle j\rangle} \\
\sum_{j=1}^{N} \ln R_{i}^{\langle j\rangle} \ln s_{i}^{\langle j\rangle}
\end{array}\right\},
$$

where $\mathrm{j}$ - the number of numerical experiment; $\mathrm{N}$ - number of experimental points.

As a result of solving equation (12), statements for estimation of parameters of regressive model take the following form: 


$$
c_{i}=\exp \left[\begin{array}{c}
\frac{\sum_{j=1}^{N} \ln R_{i}^{\langle j\rangle} \sum_{j=1}^{N} \ln R_{i}^{\langle j\rangle} \ln s_{i}^{\langle j\rangle}-\sum_{j=1}^{N} \ln ^{2} R_{i}^{\langle j\rangle} \sum_{j=1}^{N} \ln s_{i}^{\langle j\rangle}}{\left(\sum_{j=1}^{N} \ln R_{i}^{\langle j\rangle}\right)^{2}-N \sum_{j=1}^{N} \ln ^{2} R_{i}^{\langle j\rangle}} \\
n_{i}=\frac{\sum_{j=1}^{N} \ln R_{i}^{\langle j\rangle} \sum_{j=1}^{N} \ln s_{i}^{\langle j\rangle}-N \sum_{j=1}^{N} \ln R_{i}^{\langle j\rangle} \ln s_{i}^{\langle j\rangle}}{\left(\sum_{j=1}^{N} \ln R_{i}^{\langle j\rangle}\right)^{2}-N \sum_{j=1}^{N} \ln ^{2} R_{i}^{\langle j\rangle}} .
\end{array}\right.
$$

Taking into account the statement (9) for displaced points of the supporting surface it could be determined the coefficient $\mathrm{k}_{\mathrm{i}}$ of the rigidity of support as a relation of the reaction of support $\delta \mathrm{R}_{\mathrm{i}}$ to variation of displacement $\delta \mathrm{s}_{\mathrm{i}}$ :

$$
k_{i}=\frac{\delta R_{i}}{\delta s_{i}}=\frac{\delta R_{i}}{c_{i} n_{i} R_{i}^{n_{i}-1} \delta R_{i}}=\frac{R_{i}}{n_{i} s_{i}} .
$$

For clamping chart according to three planes $\mathrm{i}=1,2$, and the corresponding coefficients of rigidity for group of supports $1,2,3-\mathrm{k}_{1}$; for supports $4,5,6-\mathrm{k}_{2}$.

\section{Dynamic analysis of the system "fixture - workpiece"}

\subsection{Dynamical equation}

Consider the space movement of the workpiece, calculation scheme of which is presented on the Fig. 2.

Displacement of $\mathrm{x}_{\mathrm{i}}, \mathrm{y}_{\mathrm{i}}, \mathrm{z}_{\mathrm{i}}$ of the supporting surfaces of workpiece in the fixture regarding the stationary location, that is characterized by static displacement, determined as a result of static calculation, could be expressed by geometric relation through 6 independent parameters - degree of freedom of workpiece (Fig. 3):

- components of displacement of the center of weights $\mathrm{x}_{\mathrm{C}}, \mathrm{y}_{\mathrm{C}}, \mathrm{z}_{\mathrm{C}}$;

- rotation angles $\varphi, \psi, \theta$ of three mutually perpendicular planes $\mathrm{y}-\mathrm{z}, \mathrm{x}-\mathrm{z}, \mathrm{x}-\mathrm{y}$ around axes $\mathrm{x}, \mathrm{y}, \mathrm{z}$ of the local coordinate system, which pass through the center of the weight of workpiece $\mathrm{C}$ :

$$
x_{i}=x_{C}-\Delta x_{i}^{\prime}+\Delta x_{i}^{\prime \prime} ; \quad y_{i}=y_{C}+\Delta y_{i}^{\prime}+\Delta y_{i}^{\prime \prime \prime} ; \quad z_{i}=z_{C}+\Delta z_{i}^{\prime \prime}+\Delta z_{i}^{\prime \prime \prime},
$$

where $\Delta x i$ /, $\Delta x i / /, \Delta y i /, \Delta y i / /, \Delta z i / /, \Delta z i / / /$ additional displacements of supports through the rotation angles $\varphi, \psi, \theta$, determined by trigonometric ratios: 


$$
\begin{aligned}
& \Delta x_{i}^{\prime}=b_{i} \sin \theta+l_{i}(1-\cos \theta) ; \quad \Delta y_{i}^{\prime}=l_{i} \sin \theta-b_{i}(1-\cos \theta) ; \\
& \Delta x_{i}^{\prime \prime}=h_{i} \sin \varphi-l_{i}(1-\cos \varphi) ; \quad \Delta z_{i}^{\prime \prime}=l_{i} \sin \varphi+h_{i}(1-\cos \varphi) ; \\
& \Delta y_{i}^{\prime \prime \prime}=h_{i} \sin \psi-b_{i}(1-\cos \psi) ; \quad \Delta z_{i}^{\prime \prime \prime}=b_{i} \sin \psi+h_{i}(1-\cos \psi) .
\end{aligned}
$$

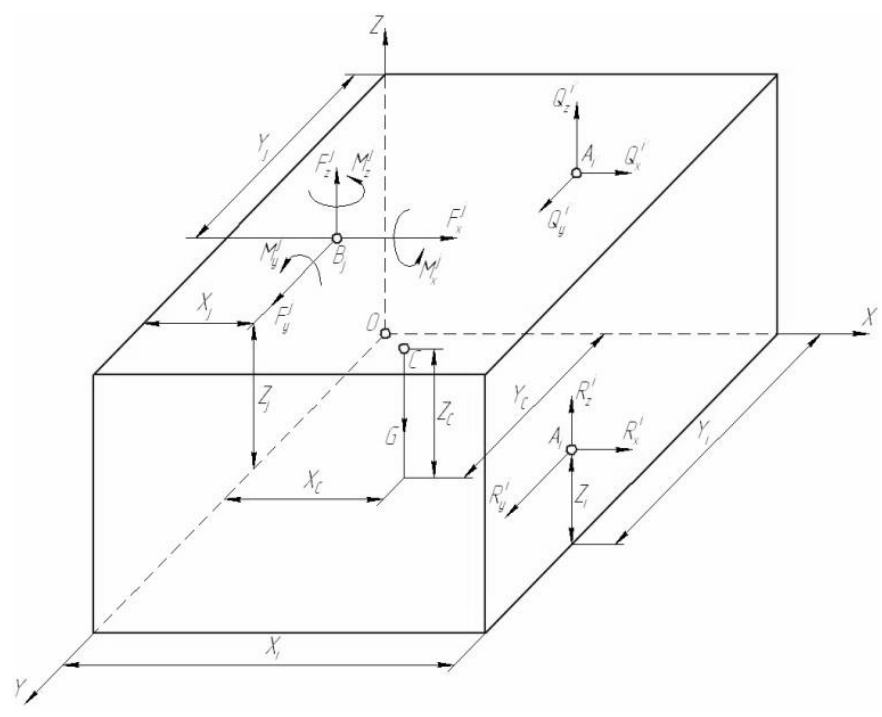

a

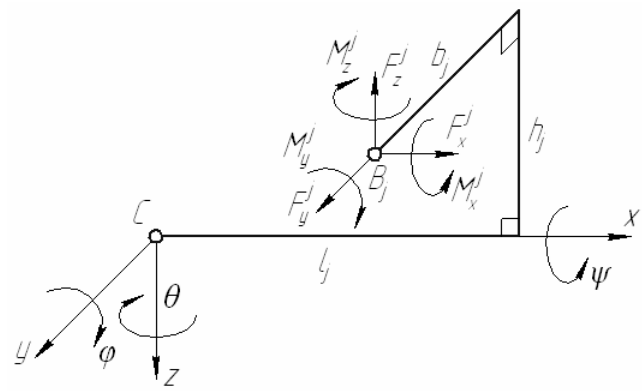

b

Fig. 2. Calculation scheme (a) and the system of dynamic components of cutting forces (b). 


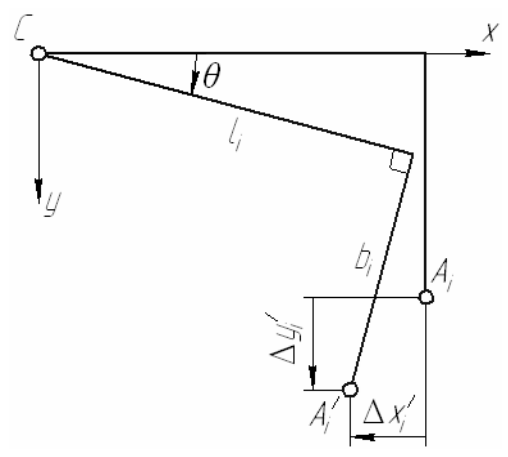

a

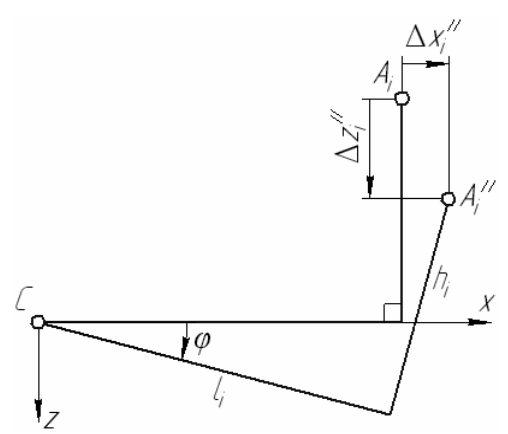

b

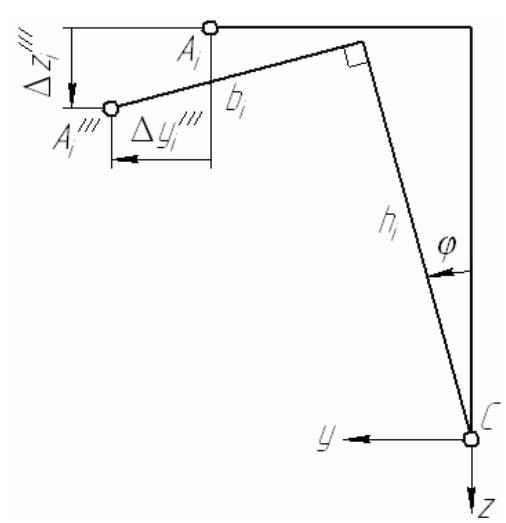

c

Fig. 3. Geometric rations for determination the additional displacements: $\Delta \mathrm{x}_{\mathrm{i}}^{\prime}, \Delta \mathrm{y}_{\mathrm{i}}^{\prime}(\mathrm{a}) ; \Delta \mathrm{x}_{\mathrm{i}}^{\prime \prime}, \Delta \mathrm{z}_{\mathrm{i}}^{\prime \prime}(\mathrm{b}) ; \Delta \mathrm{y}_{\mathrm{i}}^{\prime \prime \prime}, \Delta \mathrm{z}_{\mathrm{i}}^{\prime \prime \prime}(\mathrm{c})$. 
Parameters $\mathrm{l}_{\mathrm{i}}, \mathrm{b}_{\mathrm{i}}, \mathrm{h}_{\mathrm{i}}$ are local coordinates of supports $1,2, \ldots, 6$, connected with global coordinates $\mathrm{X}_{\mathrm{i}}, \mathrm{Y}_{\mathrm{i}}, \mathrm{Z}_{\mathrm{i}}$ by the following relations:

$$
l_{i}=X_{i}-X_{C} ; \quad b_{i}=Y_{i}-Y_{C} ; \quad h_{i}=Z_{i}-Z_{C} .
$$

For relatively small values of deformation of supports the statements (16) could be expanded into a Maclaurin series, holding members of the first order of smallness:

$$
\Delta x_{i}^{\prime}=b_{i} \theta, \quad \Delta x_{i}^{\prime \prime}=h_{i} \varphi ; \quad \Delta y_{i}^{\prime}=l_{i} \theta, \quad \Delta y_{i}^{\prime \prime \prime}=h_{i} \psi ; \quad \Delta z_{i}^{\prime \prime}=l_{i} \varphi, \quad \Delta z_{i}^{\prime \prime \prime}=b_{i} \psi .
$$

Herewith, the components of displacements of supports (15) take the form:

$$
x_{i}=x_{C}-b_{i} \theta+h_{i} \varphi ; \quad y_{i}=y_{C}+l_{i} \theta+h_{i} \psi ; \quad z_{i}=z_{C}+l_{i} \varphi+b_{i} \psi .
$$

The space movement of the workpiece in the fixture could be described by means of the theorem on movement of the center of weight and the theorem on replacement of the angular momentum of mechanical system in the projections on the axis of the local coordinate system xyz:

$$
\left\{\begin{array}{l}
m \ddot{x}_{C}=\Sigma F_{s x}^{i} ; \quad m \ddot{y}_{C}=\Sigma F_{s y}^{i} ; \quad m \ddot{z}_{C}=\Sigma F_{s z}^{i} ; \\
J_{C x} \ddot{\psi}=\Sigma M_{s x}^{i} ; \quad J_{C y} \ddot{\varphi}=\Sigma M_{s y}^{i} ; \quad J_{C z} \ddot{\theta}=\Sigma M_{s z}^{i},
\end{array}\right.
$$

where $\mathrm{m}$ - weight of workpiece; $\mathrm{J}_{\mathrm{C} x}, \mathrm{~J}_{\mathrm{Cy}}, \mathrm{J}_{\mathrm{Cz}}$ - inertia moments of workpiece relative to the axis, which pass through the center of weight; $\ddot{x}_{C}, \ddot{y}_{C}, \ddot{z}_{C}$ - projections of acceleration of the center of weight on the coordinate system; $\ddot{\psi}, \ddot{\varphi}, \ddot{\theta}-$ angular acceleration of the workpiece relatively to the coordinate axes.

Components of the spring forces and their moments (Fig. 2 b) relative to the coordinate axes taking into account the relations (19) are defined by the following statements:

$$
\begin{aligned}
& \Sigma F_{s x}^{i}=-\Sigma k_{x}^{i} x_{i}=-\Sigma k_{x}^{i}\left(x_{C}-b_{i} \theta+h_{i} \varphi\right) ; \\
& \Sigma F_{s y}^{i}=-\Sigma k_{y}^{i} y_{i}=-\Sigma k_{y}^{i}\left(y_{C}+l_{i} \theta+h_{i} \psi\right) ; \\
& \Sigma F_{s z}^{i}=-\Sigma k_{z}^{i} z_{i}=-\Sigma k_{z}^{i}\left(z_{C}+l_{i} \varphi+b_{i} \psi\right) ; \\
& \Sigma M_{s x}^{i}=\Sigma\left(-F_{s y}^{i} h_{i}-F_{s z}^{i} b_{i}\right)=-\Sigma\left[k_{y}^{i} h_{i}\left(y_{C}+l_{i} \theta+h_{i} \psi\right)+k_{z}^{i} b_{i}\left(z_{C}+l_{i} \varphi+b_{i} \psi\right)\right] \\
& \Sigma M_{s y}^{i}=\Sigma\left(-F_{s x}^{i} h_{i}-F_{s z}^{i} l_{i}\right)=-\Sigma\left[k_{x}^{i} h_{i}\left(x_{C}-b_{i} \theta+h_{i} \varphi\right)+k_{z}^{i} l_{i}\left(z_{C}+l_{i} \varphi+b_{i} \psi\right)\right] \\
& \Sigma M_{s x}^{i}=\Sigma\left(F_{s x}^{i} b_{i}-F_{s y}^{i} l_{i}\right)=-\Sigma\left[-k_{x}^{i} b_{i}\left(x_{C}-b_{i} \theta+h_{i} \varphi\right)+k_{y}^{i} l_{i}\left(y_{C}+l_{i} \theta+h_{i} \psi\right)\right]
\end{aligned}
$$

Statements (21) allow presenting the equations set (20) in the following form: 


$$
\left\{\begin{array}{l}
m \ddot{x}_{C}+\Sigma k_{x}^{i} x_{C}+\Sigma k_{x}^{i} h_{i} \varphi-\Sigma k_{x}^{i} b_{i} \theta=F_{x} ; \\
m \ddot{y}_{C}+\Sigma k_{y}^{i} y_{C}+\Sigma k_{y}^{i} h_{i} \psi+\Sigma k_{y}^{i} l_{i} \theta=F_{y} ; \\
m \ddot{z}_{C}+\Sigma k_{z}^{i} z_{C}+\Sigma k_{z}^{i} b_{i} \psi+\Sigma k_{z}^{i} l_{i} \varphi=F_{z} ; \\
J_{C x} \ddot{\psi}+\Sigma k_{y}^{i} h_{i} y_{C}+\Sigma k_{z}^{i} b_{i} z_{C}+\Sigma\left(k_{y}^{i} h_{i}^{2}+k_{z}^{i} b_{i}^{2}\right) \psi+\Sigma k_{z}^{i} l_{i} b_{i} \varphi+\Sigma k_{y}^{i} l_{i} h_{i} \theta=M_{x} ; \\
J_{C y} \ddot{\varphi}+\Sigma k_{x}^{i} h_{i} x_{C}+\Sigma k_{z}^{i} l_{i} z_{C}+\Sigma k_{z}^{i} l_{i} b_{i} \psi+\Sigma\left(k_{x}^{i} h_{i}^{2}+k_{z}^{i} l_{i}^{2}\right) \varphi-\Sigma k_{x}^{i} b_{i} h_{i} \theta=M_{y} ; \\
J_{C z} \ddot{\theta}-\Sigma k_{x}^{i} b_{i} x_{C}+\Sigma k_{y}^{i} l_{i} y_{C}+\Sigma k_{y}^{i} l_{i} h_{i} \psi-\Sigma k_{x}^{i} b_{i} h_{i} \varphi+\Sigma\left(k_{x}^{i} b_{i}^{2}+k_{y}^{i} l_{i}^{2}\right) \theta=M_{z},
\end{array}\right.
$$

where $\mathrm{m}$ - weight of the workpiece; $\mathrm{J}_{\mathrm{Cx}}, \mathrm{J}_{\mathrm{Cy}}, \mathrm{J}_{\mathrm{Cz}}$ - inertia moments of workpiece relative to the axis, which pass through the center of weight; $\ddot{x}_{C}, \ddot{y}_{C}, \ddot{z}_{C}-$ projections of acceleration of the center of weight on the coordinate system; $\ddot{\psi}, \ddot{\varphi}, \ddot{\theta}-$ angular acceleration of the workpiece relatively to the coordinate axes; $F_{x}, F_{y}, F_{z}$ - dynamical components of the cutting forces (Fig. 2 a).

Thus, the mathematical model of dynamic analysis of the workpiece in fixture is described by the system of differential equations of the 12-th order relative to 6 changeable in time independent parameters $\mathrm{x}_{\mathrm{C}}, \mathrm{y}_{\mathrm{C}}, \mathrm{z}_{\mathrm{C}}, \varphi, \psi, \theta$.

\subsection{Research of free vibrations}

For the case of zero value of forces and cutting moments, the equations set (22) in matrix form takes the following form:

$$
[M]\left\{\ddot{X}_{C}\right\}+[K]\{X\}=\{0\},
$$

where $\{0\}=\{0,0, \ldots, 0\}^{\mathrm{T}}-$ zero vector-column of an external action; $\{\mathrm{X}\}=\left\{\mathrm{x}_{\mathrm{C}}\right.$, $\left.\mathrm{y}_{\mathrm{C}}, \mathrm{z}_{\mathrm{C}}, \varphi, \psi, \theta\right\}^{\mathrm{T}}-$ vector-column of desired displacement; [M], [K] - symmetric matrix of inertia and rigidity:

$$
[M]=[M]^{T}=\left[\begin{array}{cccccc}
m & 0 & 0 & 0 & 0 & 0 \\
0 & m & 0 & 0 & 0 & 0 \\
0 & 0 & m & 0 & 0 & 0 \\
0 & 0 & 0 & J_{C x} & 0 & 0 \\
0 & 0 & 0 & 0 & J_{C y} & 0 \\
0 & 0 & 0 & 0 & 0 & J_{C z}
\end{array}\right] ;
$$




$$
[K]=[K]^{T}=\left[\begin{array}{cccccc}
k_{x x} & 0 & 0 & 0 & k_{x \varphi} & -k_{x \theta} \\
0 & k_{y y} & 0 & k_{y \psi} & 0 & k_{y \theta} \\
0 & 0 & k_{z z} & k_{z \psi} & k_{z \varphi} & 0 \\
0 & k_{y \psi} & k_{z \psi} & k_{\psi \psi} & k_{\psi \varphi} & k_{\psi \theta} \\
k_{x \varphi} & 0 & k_{z \varphi} & k_{\psi \varphi} & k_{\varphi \varphi} & -k_{\varphi \theta} \\
-k_{x \theta} & k_{y \theta} & 0 & k_{\psi \theta} & -k_{\varphi \theta} & k_{\theta \theta}
\end{array}\right] .
$$

Rigidity matrix $[\mathrm{K}]$ includes 6 consolidated coefficients of the direct rigidity

$$
\begin{aligned}
& k_{x x}=\Sigma k_{x}^{i} ; \quad k_{y y}=\Sigma k_{y}^{i} ; \quad k_{z z}=\Sigma k_{z}^{i} ; \\
& k_{y \mu \nu}=\Sigma\left(k_{y}^{i} h_{i}^{2}+k_{z}^{i} b_{i}^{2}\right) ; \quad k_{\varphi \varphi}=\Sigma\left(k_{x}^{i} h_{i}^{2}+k_{z}^{i} l_{i}^{2}\right), \quad k_{\theta \theta}=\Sigma\left(k_{x}^{i} b_{i}^{2}+k_{y}^{i} l_{i}^{2}\right)
\end{aligned}
$$

and 9 consolidated coefficients of the cross rigidity:

$$
\begin{aligned}
& k_{x \varphi}=\Sigma k_{x}^{i} h_{i} ; k_{x \theta}=\Sigma k_{x}^{i} b_{i} ; k_{y \psi}=\Sigma k_{y}^{i} h_{i} ; \\
& k_{y \theta}=\Sigma k_{y}^{i} l_{i} ; k_{z \psi}=\Sigma k_{z}^{i} b_{i} ; k_{z \varphi}=\Sigma k_{z}^{i} l_{i} ; \\
& k_{\psi \varphi}=\Sigma k_{z}^{i} l_{i} b_{i} ; k_{\psi \theta}=\Sigma k_{y}^{i} l_{i} h_{i} ; k_{\varphi \theta}=\Sigma k_{x}^{i} b_{i} h_{i},
\end{aligned}
$$

which depend on the rigidity and locating of the supporting surfaces.

In particular, during the machining of the prismatic workpiece with the center of weight $\mathrm{C}(\mathrm{L} / 2, \mathrm{~B} / 2, \mathrm{H} / 2)$ for locating chart 3-2-1 in three planes the rigidity coefficients make

$$
\begin{aligned}
& k_{x x}=\frac{1}{2} k_{y y}=k_{2} ; \quad k_{z z}=3 k_{1} ; \quad k_{\psi \psi}=\frac{1}{2} k_{1}(B-2 a)^{2} ; \\
& k_{\varphi \varphi}=\frac{3}{4} k_{1}(L-2 a)^{2} ; \quad k_{\theta \theta}=\frac{1}{2} k_{2}(L-2 a)^{2} ; \quad k_{z \varphi}=-\frac{1}{2} k_{1}(L-2 a),
\end{aligned}
$$

and another 8 coefficients of the cross rigidity make zero.

In order to consider the rigidity of the cutting tool and clamping element, the coefficient of rigidity of the clamping element $\mathrm{k}_{3}$ should be taken into account, and also the coefficients of longitudinal (along the axis z), transverse (along the axes $x, y$ ) and angular (around the axis z) rigidity of the cutting tool (an example is end mills), which could be roughly estimated by the following approximate dependencies that determine the appropriate state of stress (compression, bending and torsion):

$$
k_{p}=\frac{\pi d^{2} E}{4 L_{p}} ; \quad k_{p}^{\prime}=\frac{3 \pi d^{4} E}{32 L_{p}^{3}} ; \quad k_{p}^{\prime \prime}=\frac{\pi d^{4} E}{64(1+v) L_{p}},
$$

where $\mathrm{d}, \mathrm{L}_{\mathrm{p}}$ - diameter and length of the mill; E, $v$ - elasticity modulus of the first grade and Poisson's ratio of mill's material. 
The consolidated coefficients of the direct and cross rigidities with the consideration of rigidities of the cutting instruments and clamping device receive the following form:

$$
\begin{aligned}
& k_{x x}=k_{2}+k_{p}^{\prime} ; \quad k_{y y}=2 k_{2}+k_{p}^{\prime} ; \quad k_{z z}=3 k_{1}+k_{3} ; \\
& k_{\psi \psi}=\frac{1}{2} k_{1}(B-2 a)^{2}+k_{p}\left(\frac{B}{2}-Y\right)^{2}+k_{p}^{\prime}\left(H_{1}-\frac{H}{2}\right)^{2} ; \\
& k_{\varphi \varphi}=\frac{3}{4} k_{1}(L-2 a)^{2}+k_{p}\left(\frac{L}{2}-X\right)^{2}+k_{p}^{\prime}\left(H_{1}-\frac{H}{2}\right)^{2} ; \\
& k_{\theta \theta}=\frac{1}{2} k_{2}(L-2 a)^{2}+k_{p}^{\prime}\left[\left(\frac{L}{2}-X\right)^{2}+\left(\frac{B}{2}-Y\right)^{2}\right]+k_{p}^{\prime \prime} ; \\
& k_{x \varphi}=-k_{p}^{\prime}\left(H_{1}-\frac{H}{2}\right) ; k_{x \theta}=-k_{p}^{\prime}\left(\frac{B}{2}-Y\right) ; k_{y \psi}=k_{p}^{\prime}\left(H_{1}-\frac{H}{2}\right) ; \\
& k_{y \theta}=-k_{p}^{\prime}\left(\frac{L}{2}-X\right) ; k_{z \psi}=-k_{p}\left(\frac{B}{2}-Y\right) ; k_{z \varphi}=-\frac{1}{2} k_{1}(L-2 a)-k_{p}\left(\frac{L}{2}-X\right) ; \\
& k_{\psi \varphi}=k_{p}\left(\frac{L}{2}-X\right)\left(\frac{B}{2}-Y\right) ; k_{\psi \theta}=-k_{p}^{\prime}\left(\frac{L}{2}-X\right)\left(H_{1}-\frac{H}{2}\right) ; \\
& k_{\varphi \theta}=-k_{p}^{\prime}\left(\frac{B}{2}-Y\right)\left(H_{1}-\frac{H}{2}\right) .
\end{aligned}
$$

During the cutting process, the presented coefficients of rigidity change according to the value, among them 4 - change its sign as a result of change of the coordinate $\mathrm{Y}$ of the location of cutting tool.

For free vibrations of the mechanical system "fixture - workpiece", the solution of the equation (23) is a harmonic movement described by the following statement:

$$
\{X\}=\left\{X_{a}\right\} \sin \omega t,
$$

which includes an unknown own frequency $\omega$, and also vector-column of the peak values of displacements and rotation angles around the local system of coordinates $\left\{\mathrm{X}_{\mathrm{a}}\right\}=\left\{\mathrm{x}_{\mathrm{Ca}}, \mathrm{y}_{\mathrm{Ca}}, \mathrm{z}_{\mathrm{Ca}}, \varphi_{\mathrm{a}}, \psi_{\mathrm{a}}, \theta_{\mathrm{a}}\right\}^{\mathrm{T}}$. The last determines the form of free system vibrations with the accuracy up to the constant multiplier.

Equation (23) with the consideration of the statements (31) receives the following form:

$$
\left([K]-\omega^{2}[M]\left(X_{a}\right)=\{0\}\right.
$$

The condition of existing of the nontrivial solutions of equation (32) allows recording the frequency equation:

$$
\left|[K]-\omega^{2}[M]\right|=0 .
$$


from which 6 free frequencies of the mechanical system are determined:

$$
\begin{aligned}
& \omega_{1}=0 \sqrt{\frac{k_{2}}{m}} ; \quad \omega_{2}=\sqrt{\frac{2 k_{2}}{m}} ; \\
& \omega_{3}=\sqrt{\frac{3 k_{1}}{2}\left\{\frac{1}{m}+\frac{(L-2 a)^{2}}{4 J_{C y}}-\sqrt{\left[\frac{1}{m}+\frac{(L-2 a)^{2}}{4 J_{C y}}\right]^{2}-\frac{8(L-2 a)^{2}}{9 m J_{C y}}}\right\}} \\
& \omega_{4}=(B-2 a) \sqrt{\frac{k_{1}}{2 J_{C x}}} ; \\
& \omega_{5}=\sqrt{\frac{3 k_{1}}{2}\left\{\frac{1}{m}+\frac{(L-2 a)^{2}}{4 J_{C y}}+\sqrt{\left[\frac{1}{m}+\frac{(L-2 a)^{2}}{4 J_{C y}}\right]^{2}-\frac{8(L-2 a)^{2}}{9 m J_{C y}}}\right\}} ; \\
& \omega_{6}=(L-2 a) \sqrt{\frac{k_{2}}{2 J_{C z}}} .
\end{aligned}
$$

the $3^{\text {rd }}$ and the $5^{\text {th }}$ free frequencies ( $\omega_{n} \in$ Re $)$ with consideration of the formula (34) obtain the real values during performing the following inequality:

$$
\left[\frac{1}{m}+\frac{(L-2 a)^{2}}{4 J_{C y}}\right]^{2}>\frac{8(L-2 a)^{2}}{9 m J_{C y}} .
$$

Introduction of the dimensionless parameter

$$
\alpha=\frac{m(L-2 a)^{2}}{J_{C y}},
$$

which characterizes the relation of the inertia moments of workpiece, allow reducing inequality (35) after the identic transformations to the simplest form:

$$
\frac{\alpha^{2}}{16}-\frac{7 \alpha}{18}+1>0,
$$

which is always performed.

Under condition (33) the equation (32) has the nontrivial solutions, which determine 6 forms of free vibrations with the accuracy up to the constant multiplier: 


$$
\left\{\begin{array}{l}
y_{C}^{\langle n\rangle} \\
z_{C}^{\langle n\rangle} \\
\varphi^{\langle n\rangle} \\
\psi^{\langle n\rangle} \\
\theta^{\langle n\rangle}
\end{array}\right\}=-\left[d_{i, j}^{\langle n\rangle}\right]\left\{\begin{array}{l}
K_{2,1}-\omega_{n}^{2} M_{2,1} \\
K_{3,1}-\omega_{n}^{2} M_{3,1} \\
K_{4,1}-\omega_{n}^{2} M_{4,1} \\
K_{5,1}-\omega_{n}^{2} M_{5,1} \\
K_{6,1}-\omega_{n}^{2} M_{6,1}
\end{array}\right\},
$$

where $\left[d_{i, j}^{\langle n\rangle}\right]$ - the main diagonal minor of the matrix of dynamic rigidity [D], which elements

$$
d_{i, j}^{\langle n\rangle}=K_{i, j}-\omega_{n} M_{i, j}, \quad(i, j=2,3, \ldots, 6) .
$$

are determined for the definite free frequency $\omega_{\mathrm{n}}(\mathrm{n}=1,2, \ldots, 6)$.

\subsection{Research of the forced vibrations}

Spatial motion of the workpiece in fixture as a body with 6 degrees of freedom in projections on the axes of the local system of coordinate xyz has the following form of equation (23), which could be presented in matrix form:

$$
[M]\left\{\ddot{X}_{C}\right\}+[K]\{X\}=\{F\},
$$

where $\{\mathrm{X}\}$ - vector-column of an unknown displacements; $\{\mathrm{F}\}$ - vector-column of external dynamical action:

$$
\{X\}=\left\{\begin{array}{c}
x_{C} \\
y_{C} \\
z_{C} \\
\varphi \\
\psi \\
\theta
\end{array}\right\} ; \quad\{F\}=\left\{\begin{array}{c}
F_{x} \\
F_{y} \\
F_{z} \\
M_{x} \\
M_{y} \\
M_{z}
\end{array}\right\} .
$$

Herewith, the moments of dynamical components of cutting forces regarding axes of the local system of coordinates (fig. 2 b):

$$
\begin{aligned}
& M_{x}=F_{y} h_{A}+F_{z} b_{A} ; \\
& M_{y}=F_{x} h_{A}+F_{z} l_{A} ; \\
& M_{z}=-F_{x} b_{A}+F_{y} l_{B} .
\end{aligned}
$$

Consider the case of monoharmonic external action:

$$
\{F\}=\left\{F_{a}\right\} \sin \omega t,
$$


where $\left\{F_{a}\right\}$ - vector-column of amplitudes of external forces and moments; $\omega=\omega_{0} \mathrm{Z}-$ frequency, which is equal to the product of rotation frequency of the spindle and number of teeth $\mathrm{z}$ of the cutting tool.

Solution of equation (40) is sought in the form

$$
\{X\}=\left\{X_{a}\right\} \sin \omega t .
$$

For vector-column $\left\{X_{a}\right\}$, elements of which are displacement amplitudes of center of weight and rotation angles around the axes of coordinates.

Substitution of the statement (44) into the equation (40) allows writing down the equation

$$
\left([K]-\omega^{2}[M]\right)\left\{X_{a}\right\}=\left\{F_{a}\right\},
$$

the solution of which is

$$
\left\{X_{a}\right\}=\left([K]-\omega^{2}[M]\right)^{-1}\left\{F_{a}\right\} .
$$

Dependence of the component $X_{a i}$ of vector-column $\left\{X_{a}\right\}$ on frequency of external action $\omega$ determines the frequency-response characteristics of the mechanical system «fixture - workpiece».

For locating chart 3-2-1 according to 3 planes frequency values of displacements of the center of weight of workpiece and rotation angles around the axes of coordinates have the following form:

$$
\begin{aligned}
& x_{C a}(\omega)=\frac{F_{x a} / m}{\omega_{1}^{2}-\omega^{2}} ; y_{C a}(\omega)=\frac{F_{y a} / m}{\omega_{2}^{2}-\omega^{2}} ; z_{c a}(\omega)=\frac{\left(\frac{k_{\varphi \varphi}}{J_{C y}}-\omega^{2}\right) \frac{F_{z a}}{m}-\frac{k_{z \varphi}}{J_{C y}} \frac{M_{z a}}{m}}{\left(\omega_{3}^{2}-\omega^{2}\right)\left(\omega_{5}^{2}-\omega^{2}\right)} ; \\
& \psi_{a}(\omega)=\frac{M_{x a} / J_{C x}}{\omega_{4}^{2}-\omega^{2}} ; \varphi_{a}(\omega)=\frac{-\frac{k_{z \varphi}}{J_{C y}} \frac{F_{z a}}{m}+\left(\frac{k_{z z}}{m}-\omega^{2}\right) \frac{M_{z a}}{J_{C y}}}{\left(\omega_{3}^{2}-\omega^{2}\right)\left(\omega_{5}^{2}-\omega^{2}\right)} ; \theta_{a}(\omega)=\frac{M_{z a} / J_{C z}}{\omega_{6}^{2}-\omega^{2}},
\end{aligned}
$$

where $\omega_{1}, \omega_{2}, \ldots, \omega_{6}-$ value of free frequencies, determined as a result of modal analysis.

According to the formula of passing from displacement of the center of weight of workpiece to displacement of supports

$$
x_{i}=x_{C}-b_{i} \theta+h_{i} \varphi ; \quad y_{i}=y_{C}+l_{i} \theta+h_{i} \psi ; \quad z_{i}=z_{C}+l_{i} \varphi+b_{i} \psi
$$

the frequency-response characteristics for supporting points have the following form: 


$$
\begin{aligned}
& x_{i a}=\frac{F_{x a} / m}{\omega_{1}^{2}-\omega^{2}}+h_{i} \frac{-\frac{k_{z \varphi}}{J_{C y}} \frac{F_{z a}}{m}+\left(\frac{k_{z z}}{m}-\omega^{2}\right) \frac{M_{z a}}{J_{C y}}}{\left(\omega_{3}^{2}-\omega^{2}\right)\left(\omega_{5}^{2}-\omega^{2}\right)}-b_{i} \frac{M_{z a} / J_{C z}}{\omega_{6}^{2}-\omega^{2}} ; \\
& y_{i a}=\frac{F_{y a} / m}{\omega_{2}^{2}-\omega^{2}}+h_{i} \frac{M_{x a} / J_{C x}}{\omega_{4}^{2}-\omega^{2}}+l_{i} \frac{M_{z a} / J_{C z}}{\omega_{6}^{2}-\omega^{2}} ; \\
& z_{i a}=\frac{\left(\frac{k_{\varphi \varphi}-k_{z \varphi} l_{i}}{J_{C y}}-\omega^{2}\right) \frac{F_{z a}}{m}+\left(\frac{k_{z z}-k_{z \varphi} / l_{i}}{m}-\omega^{2}\right) \frac{M_{z a} l_{i}}{J_{C y}}}{\left(\omega_{3}^{2}-\omega^{2}\right)\left(\omega_{5}^{2}-\omega^{2}\right)}+b_{i} \frac{M_{x a} / J_{C x}}{\omega_{4}^{2}-\omega^{2}} .
\end{aligned}
$$

So, for locating chart 3-2-1 according to three planes the location of mill $\mathrm{Y}=\mathrm{B} / 2$ the frequency-response characteristics of the system "fixture - workpiece" in the bottom supporting points have the following form:

$$
\begin{aligned}
& z_{1 a}=z_{2 a}=\frac{0,5 k_{1}(L-2 a)^{2}-J_{C y} \omega^{2}}{\left(\omega_{3}^{2}-\omega^{2}\right)\left(\omega_{5}^{2}-\omega^{2}\right)} \frac{F_{z a}}{m J_{C y}} ; \\
& z_{3 a}=\frac{k_{1}(L-2 a)^{2}-J_{C y} \omega^{2}}{\left(\omega_{3}^{2}-\omega^{2}\right)\left(\omega_{5}^{2}-\omega^{2}\right)} \frac{F_{z a}}{m J_{C y}} .
\end{aligned}
$$

The analysis of the last dependencies confirms that frequencies

$$
\omega^{\prime}=(L-2 a) \sqrt{\frac{k_{1}}{2 J_{C y}}} ; \quad \omega^{\prime \prime}=(L-2 a) \sqrt{\frac{k_{1}}{J_{C y}}}=\frac{\omega^{\prime}}{\sqrt{2}} ;
$$

correspond the appearance of the antiresonance effect, for which zero value of amplitudes $\mathrm{z}_{1 \mathrm{a}}, \mathrm{z}_{2 \mathrm{a}}, \mathrm{i} \mathrm{z}_{3 \mathrm{a}}$ are is observed.

\section{Conclusions}

The study of the previous experience, generalization and systematization of the existing approaches to the research of mechanical systems allowed making a proposal on development a new approach, which is the comprehensive analysis of equilibrium state and vibrations relatively fixed position of the workpiece as an element of the mechanical system with taking into account the rigidity of the cutting tool.

The mathematical model of static calculation system "fixture - workpiece" is being proposed, which ensures the defining of contact points of functional elements of fixture with the workpiece, the minimum required clamping force, components of cutting forces and places of application of these forces. Regression dependences for estimation of rigidity of functional elements of machine devices are being proposed.

The mathematical model of dynamic analysis, which is described by the system of differential equations of the 12 orders in matrix form relatively 6 variables in time independent parameters has been developed. The presented model allows research the 
free frequencies of the system "fixture - workpiece" and determines the appropriate forms of free vibrations. The model also allows research the forced vibrations of the workpiece relatively the balanced position as a result of the action of dynamic component forces and cutting moments, and also determines frequency-response characteristics and the frequencies of resonance and antiresonance appearance.

The following researches are directed on the development of the methodology of calculation of the system "fixture - workpiece" by computer means of numerous realization and development of practical recommendations regarding its use during fixture design.

\section{References}

1. Karpus, V.E., Ivanov, V.O., Kotliar, O.V., Minenko, D.O., Ivanova, M.S.: Intensification of Manufacturing Processes. Sumy State University, Sumy (2012), doi: 10.13140/2.1.3953.7604 [in Ukrainian].

2. Bi, Z.M., Zhang, W.J.: Flexible Fixture Design and Automation: Review, Issues and Future Directions. International Journal of Production Research, Vol. 39, pp. 2867-2894 (2001), doi: 10.1080/00207540110054579.

3. Ivanov, V.: Choice of Optimal Fixtures for CNC Machine Tools. Ph.D. Thesis. National Technical University "Kharkiv Polytechnic Institute", Kharkiv, Ukraine (2010) [in Ukrainian].

4. Nixon, F.: Managing to Achieve Quality and Reliability. McGraw Hill, Maidenhead (1971).

5. Rong, Y., Zhu, Y.: Computer-Aided Fixture Design. Marcel Dekker, New York (1999).

6. Kumbhar, N., Patil, G., Mohite, S., Sutar M.: Finite Element Modelling and Analysis of Workpiece-Fixture System. International Journal of Applied Research in Mechanical Engineering, Vol. 2, Issue 2, pp. 60-65 (2012).

7. Liao, Y., Hu, S.: An Integrated Model of a Fixture-Workpiece System for Surface Quality Prediction. International Journal of Advanced Manufacturing Technology, Vol. 17, Issue 11, pp. 810-818 (2001), doi: 10.1007/s001700170108.

8. Kang, Y., Rong, Y., Yang, J.C.: Computer-Aided Fixture Design Verification. Part 3. Stability Analysis. International Journal of Advanced Manufacturing Technologies, Vol. 21, Issue 10, pp. 842-849 (2003), doi: 10.1007/s00170-002-1401-4.

9. Asante, J. N.: Effect of Fixture Compliance and Cutting Conditions on Workpiece Stability. International Journal of Advanced Manufacturing Technology, Vol. 48, Issue 1, pp. 33-43 (2010), doi: 10.1007/s00170-009-2284-4.

10. Cioata, V., Kiss, I.: The Machining Error due to Contact Deformation of Workpiece-Fixture System. Acta Technica Corviniensis - Bulletin of Engineering, Vol. 2, Issue 1, pp. 33-36 (2009).

11. Zheng, Y.: Finite Element Analysis for Fixture Stiffness. PhD Thesis. Worcester Polytechnic Institute, Worcester, MA, USA (2005).

12. Asada, H., By, A.: Kinematic Analysis of Workpart Fixturing for Flexible Assembly with Automatically Reconfigurable Fixtures. IEEE Journal on Robotics and Automation, Vol. 1, Issue 2, pp. 86-94 (1985), doi: 10.1109/JRA.1985.1087007.

13. Rong, Y., Bai, Y.: Automated Generation of Fixture Configuration Design. Journal of Manufacturing Science and Engineering, Vol. 119, Issue 2, pp. 208-219 (1997), doi: $10.1115 / 1.2831097$. 
14. Chou, Y.C.: Automated Fixture Design for Concurrent Manufacturing Planning. Concurrent Engineering, Vol. 1, Issue 4, pp. 219-229 (1993), doi: 10.1177/1063293X9300100405.

15. Wu, Y., Rong, Y., Chu, T.: Automated Generation of Dedicated Fixture Design. International Journal Computer Application in Technologies, Vol. 10, Issues 3-4, pp. 213-235 (1997), doi: 10.1504/IJCAT.1997.062249.

16. Trappey, A.J.C., Su, C.S., Hou, J.L.: Computer-Aided Fixture Analysis Using Finite Element Analysis and Mathematical Optimization Modeling. Journal of Manufacturing Science and Engineering, Vol. 2-1, pp. 777-787 (1995).

17. Deng, H.: Analysis and Synthesis of Fixturing Dynamic Stability in Machining Accounting for Material Removal Effect. Ph.D. Thesis. Georgia Institute of Technology, Atlanta, GA, USA (2006).

18. Pashkevich, M.F., Pashkevich, V.M., Mironova, M.N.: Research of Workpiece Displacement Under Clamping Forces During Locating on Surface. Bulletin of Gomel State Technical University named after P.O. Suhoi, Vol. 2 (41), pp. 9-18 (2010) [in Russian].

19. Ivanov, V., Pavlenko, I., Protsai, R. Determination of Conditions of Ensuring Workpiece Stability in the Fixture. Proc. of $12^{\text {th }}$ Int. Symposium of Ukrainian Mechanical Engineers in Lviv, Lviv, Ukraine, May 28-29, 2015, pp. 84-85 (2015) [in Ukrainian].

20. Pavlenko, I.V., Ivanov V.O.: Ensuring of the Workpiece Stability Conditions in the Fixture with Locating Scheme on Three Planes. Bulletin of Sumy National Agrarian University, Vol. 11 (27), pp. 23-26 (2015) [in Ukrainian].

21. Kurylov, B.M., Pavlenko, I.V., Ivanov, V.O. Static and Dynamic Analysis of Mechanical System "Fixture - Workpiece". Proc. of $4^{\text {th }}$ All-Ukr. Conf. Modern Technologies in Industrial Manufacturing, Sumy, Ukraine, April 19-22, 2016, Vol. 1, pp. 165-166 (2016) [in Ukrainian].

22. Ivanov, V.A., Pavlenko I.V., Kurilov, B.N. et al.: Harmonic Analysis of the System "Fixture - Workpiece" with Locating Scheme on Three Planes. Proc. of Int. Conf. Innovations in Machining: Opinion of Young Specialists, Kursk, Russia, October 2-3, 2015, pp. 158161 (2015) [in Russian].

23. Pavlenko, I.V., Ivanov, V.A., Kurilov B.N. et al.: Mathematical Model of the Dynamics of Mechanical System "Fixture - Workpiece" with Locating Scheme on Three Planes. Proc. of Int. Conf. Safety and Engineering Design in Manufacturing Engineering, Kursk, Russia, October 25-26, 2015, pp. 119-122 (2015) [in Russian].

24. Ivanov, V.O., Pavlenko, I.V. Estimation of Nonlinear Stiffness of Functional Elements of Fixtures. Proc. of $16^{\text {th }}$ Int. Conf. Manufacturing of Ukraine: Progressive Ideas - Science Manufacturing, Sumy, Ukraine, October 26-29, 2016, pp. 23-24 (2016) [in Ukrainian].

25. Kurylov, B.M., Pavlenko, I.V., Ivanov, V.O.: Using Computer Applications for Investigation the Dynamics of Mechanical System "Fixture - Workpiece". Proc. of Conf. Modern Technologies in Industrial Manufacturing, Sumy, Ukraine, April 18-21, 2017, Vol. 1, p. 149 (2017) [in Ukrainian].

26. Karpus, V.E., Ivanov, V.O., Minenko, D.O., Dehtiarov, I.M.: Rapid-adjustable locating modules for prismatic parts. New Materials and Technologies in Metallurgy and Manufacturing, Vol. 2, pp. 91-94 (2012) [in Ukrainian]. 